\title{
AN EFFICIENT DENSITY PARAMETER-LIGHT IN ENHANCED SUBSPACE CLUSTERING IN HIGH DIMENSIONAL DATA
}

\author{
Rama Devi Jujjuri ${ }^{1}$ and M.Venkateswara Rao ${ }^{2}$ \\ ${ }^{1}$ Department of Computer Science and Engineering, Gandhi Institute of \\ Technology and Management (GITAM), Visakhapatnam, India \\ ${ }^{2}$ Department of Information Technology Gandhi Institute of Technology and \\ Management (GITAM), Visakhapatnam, India \\ ${ }^{1}$ ramadevi@pvpsiddhartha.ac.in, ${ }^{2}$ mandapati9venkat@gmail.com
}

\begin{abstract}
Subspace clustering identifies the clusters stored in subspaces of a high dimensional dataset. Various Density-based strategies have been determined to mine clusters of arbitrary shape successfully even in the appearance of noise in full dimensional space clustering techniques. The performance and result of a subspace clustering algorithm highly depend on the parameter values of the algorithm is tuned to execute. Although determining the proper parameter values are crucial for both clustering quality and performance of the algorithm. Further, as high dimensional data has converted more and more prevalent in real-world applications due to the advances of vast data technologies. Precisely Density-based subspace clustering have gained their importance owing to their ability to identify arbitrary shaped subspace clusters. Density Divergence Query is an essential subject in high dimensional data clustering. Density divergence involves having various subspace cardinalities for complex region densities. To defeat this problem, EfficientEnSubClu employs an efficient subspace clustering model. It discovers the clusters using different epsilon density thresholds in various subspaces. In this research, we propose an Efficient enhanced Subspace Clustering Model named EfficientEnSubClu (Enhancement of ENSUBCLU) for discovering precise values of parameters in subspace clustering. It allows efficient neighboring core points to be clustered and find quality subspace clusters satisfying specific qualitative and quantitative properties.

Furthermore, apply the post-processing clustering steps on each found subspaces. It aims a merging model at the first step of evaluation of clusters connected with DBSCAN algorithm. Also, find the number of subspace clusters in a particular dimension and calculate the low mean dimensionality of subspace clusters. It represents every cluster with a fewer number of dimensions as visible from the low utility of mean dimensionality. Hence, we can obtain knowledge more concisely with an enhanced quality of clusters in terms like Accuracy and Silhouette Coefficient.
\end{abstract}

Keywords - Subspace Clustering; Density divergence; Parameter epsilon; High Dimensional Data Mining; Density based Subspace Clustering; Quality Subspace Clusters 


\section{INTRODUCTION}

Clustering is a significant chore that employed different applicability. Clusters may survive under various subspaces of multidimensional data. Popular full dimensional space clustering algorithms [1] have complexity in recognizing these clusters. Subspace clustering algorithms have applied different subspace search approaches. Being a result, the subspace selection and clustering processes tightly coupled. Subspace clustering has been proposed to overcome the problems in traditional clustering faced in datasets with a large number of attributes. Subspace clustering research [2] has been collecting research field. Essential subspace clustering techniques concentrate the data which are rigorously collective in their subspaces. The three essential alternatives are the grid-based, density based and window based approaches.

Although these schemes are useful in solving their clustering difficulties, their limitations exist delivered by the recent proliferation of complex data and the need for higher quality clustering effects. Certain fuels the research in enhanced subspace clustering [3,4]. Enhanced subspace clustering can be categorized by two associations, Handling complex data and improving clustering results. In Handling complex data the basic subspace clustering methods only manage quantitative two-dimensional (2D) data (object $\times$ attribute) and do not handle complex data of higher order 3D data (object $\times$ attribute $\times$ time), extensive streaming data, noisy data or categorical data. Improving clustering results undergoes from the shortcomings of basic subspace clustering methods, which can be broadly categorized. First, mine the significant subspace quality clusters in the high dimensional data. Second, mine the input parameters in the popular algorithms need the user to initiate tuning parameters and clusters. These techniques are sensitive to specific tuning parameters and maintained that tuning parameters are not significant and challenging for the user to set the right parameter contexts. This situation increased as conventional algorithms are overflowed with tuning parameters and involving the clustering task. Accordingly, it is desirable to overcome this parameter-sensitivity problem of the existing methods. Though choosing suitable parameter values is not a simple job, for prior knowledge is not reasonable in common. One reasonable approach may be a trial-and-error system, which frequently works clustering efficiencies among various series of input parameter values and then subsequently takes the most satisfying outcome.

Although, this method follows: as clustering is truly a computation-intensive task, its running time is typically long, so trying many of series of parameters may not be achievable. Improve the clustering results in a variety of ways, from working significant subspace clusters to implementing parameter-insensitive clustering techniques. In parameter-insensitive clustering, the 'real' subspace clusters identified, and they are not ideas of skewed parameter contexts.

Most of the subspace clustering techniques adopt density-based clustering methods. The natural clusters are identified using density-based clustering techniques as they do not require the number of clusters as an input parameter. These algorithms are also insensitive to outliers. DBSCAN is the density-based clustering algorithms which use two input parameters, namely min points and epsilon to obtain density connectivity with the objects and form dense clusters [5]. Density-based Subspace Clustering algorithms have picked up their significance inferable from their ability to observe arbitrary shaped subspace clusters. DBSCAN produces excellent results with appropriate input parameters. DBSCAN mainly used in ENSUBCLU, which is a successful subspace clustering algorithm. ENSUBCLU applies DBSCAN to detect clusters in $(\mathrm{k}+1)$-dimensional subspace by further partitioning the objects of clusters recognized in k-dimensional subspaces [6]. 


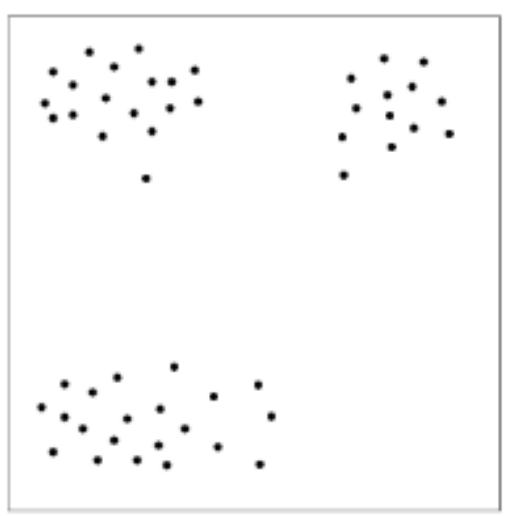

Fig. 1 Uniform spread of data objects in a Subspace

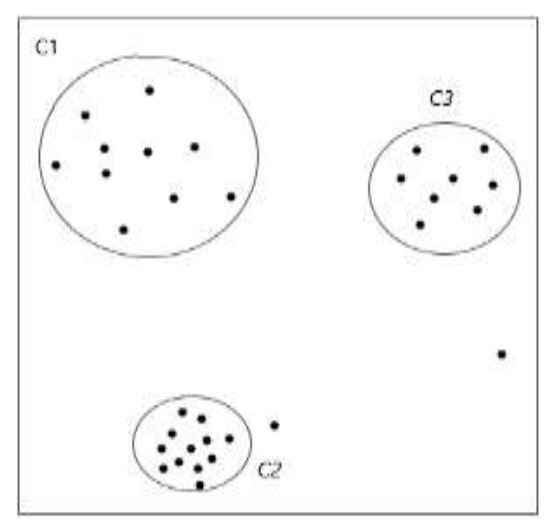

Fig. 2 Non- Uniform spread of data ob Subspace

Due to the concept of density divergence, the objects in subspaces of higher dimensions are expected to be spread farther away, which calls for subspace specific parameter setting [7-8]. Here, authors suggest an algorithm to handle density divergence of datasets by selecting the relevant values of epsilon dynamically by the spread of the data objects in the respective subspaces. In this algorithm, the authors propose to dynamically compute epsilon for each subspace depending on the spread of the data in the subspace and use it for identifying the density connected subspace clusters in the second phase which is specific to deal with subspaces containing clusters with various densities.

Section II described recent developments on Density-based subspace clustering and enhanced subspace clustering algorithms. Section III describes the proposed algorithm that handles density divergence problem and applies the post-processing steps on each found subspaces and computes the low dimensionality of subspace clusters retrieved by Efficient-EnSubClu. The experimental results and analysis specified in Section IV. Section $\mathrm{V}$ determines the paper was producing conclusion and additional extensions.

\section{RELATED WORK}

Clustering- In Quest (CLIQUE) is one of the underlying grid based subspace clustering algorithm. It partitions the data space into equal-sized grids and finds the one whose density exceeds a fixed density threshold being dense regions in the subsets. The higher dimensional dense grids are found based on apriori style of pruning ENtropy based subspace CLUStering calls ENCLUS makes use of the concept of entropy in obtaining dense regions. It is a continuation for CLIQUE that mines maximum correlated subspaces which have more clustering performance of the data objects. Using downward and upward closure features given by entropy and dimensional correlation, respectively, the algorithm was able to mine the non-minimal correlated subspaces. Merging of Adaptive Finite Intervals called MAFIA[9] uses the technique of adaptive grids based on the data distribution. The identification of higher dimensional subspace clusters is the same as that of CLIQUE[10]. These traditional algorithms suffer from the problem of density divergence. The Grid-based subspace clustering algorithms result in poor quality clusters if there is no proper positioning of the grids. So the maximum of the research is going on to relieve this problem Density via-based subspace clustering algorithms. Density Conscious Subspace clustering is called DENCOS[11], which is a grid-based subspace clustering algorithm which determines dense thresholds according to the dimensionality of the subspace. The dense region grids were identified using a data structure denoted Density FP-tree. Variable density thresholds are employed to discover dense regions in subspaces of varied dimensional sizes, the property of monotonicity no more holds. So, all the subspaces considered in exploring dense regions [11]. 
SUBCLU(density connected SUBspace CLUstering) is the constant density based subspace clustering algorithm that discards the use of grids and overwhelms the limitations of grid-based subspace clustering algorithms. Furthermore, it performs the use of density connectivity in detecting arbitrary shaped dense clusters in subspaces and follows apriori style of pruning in exploring higher dimensional subspaces [5]. It uses DBSCAN that uses the same value for the input parameters, namely epsilon and minpts in all the subspaces, thus degenerating its appearance.

Filter Refinement Subspace Clustering (FIRES) is a generic framework that uses approximate techniques in a filter-refinement method that scales with the size of the data dimensionality. The clustering method is carried based on local density threshold. By merging of the base clusters derived from one-dimensional clusters the approximations of maximal-dimensional subspace clusters are determined[12]

DUSC (Dimensionality Unbiased Subspace Clustering) uses the statistical function called Epanechnikov kernel for computing local density model adaptive to the dimensionality of the subspace clusters. The density threshold alters with the size of the dimensionality. Due to this, preventing subspaces using anti-monotonicity is not possible. It examines the problem of density divergence to some extent, but all the subspaces have to be examined for finding dense subspace clusters [13].

SSCS, Scalable density-based subspace clustering approach steers mining a some selected subspace clusters. The steering method recognizes assuring subspaces and their combinations directly for clustering - i.e., the current subspace clusters. The steering process includes adequate information collection for the density-based scheme, and the processing system needs to databases in a few subspace dense regions. It joins intermediate cluster results to ultimately recognize distinct regions for subspace clustering in a best first practice. It shows that database scans for density estimates are softened[14]. INSCY[15] is one essential index data structure is indexing subspace clusters called INSCY. In this new index, structures were used for efficient clusters and remove redundancy clusters but obtained multiple database scans. Fast and Scalable Subspace Clustering for High dimensional data called SUBSCALE[16] approach is used to find the non-trivial subspace clusters with minimal cost. Also, scales very well with the dimensionality and is highly parallelizable. However, it can't handle the density divergence problem in case of both uniform and non-uniform spread of data objects. EDENCOS [17] Efficient Density subspace clustering called EDENCOS (Efficient Density Conscious Subspace Clustering) used top-down and bottom-up approaches. The depth-first search method used in EDENCOS. It is a practical, incremental updating, FP mining method which keeps FPs efficiently. This technique follows FP-Tree with children table and trailer table to avoid the repetitions in the scanning, reconstructing and computing. However, it takes more run time for increasing of attributes in the high dimensional data sets.

Interesting Subspace Clustering (ISC) method [18] is approaching this point by applying an attribute dependency measure defined in Rough Set theory. ENSUBCLU [6] This algorithm based on density clustering paradigm and subspace steering approach is used to find the promising subspaces and different discretization points are adjusted to detect the clusters in relevant subspace cardinalities. This model reduces the number of database scans and also to eliminate random subspaces used in hash mapping collision procedure. Next merged the dense-point units and refined the clusters. ENSUBCLU proved to be efficient like SUBSCALE [16], and it replaces all other conventional subspace clustering algorithms with its features. However, they(mentioned all existing algorithms) suffer from the problem of density divergence. So the author follows the tunning the parameters inorder to improve the significance of clusters - the next section to address the parameteric free on various subspace clustering algorithms. 


\subsection{PARAMETRIC FREE ON SUBSPACE CLUSTERING ALGORITHMS}

The one crucial, challenging task in high dimensional data is Subspace clustering method. There have been various strategies proposed in the previous literature to recognize clusters in subspaces; though, their play and quality are profoundly affected by input parameters. A little analysis is done so far on understanding proper parameter values automatically - the parameter-search scheme based on determining proper values of tuning parameters. Though, their execution and quality modified with input parameters. Little research is done so far on knowing precise parameter values automatically. Selecting proper parameter values is not a simple task, for preliminary information is not available in common. The parameter-search methods are discussed based on determining proper values of tuning parameters. Impact Parameter Analysis of Subspace Clustering [19] is a parameter-search process. It follows a random sampling procedure. Conduct the experiments to present the impact of parameters in subspace clustering and to find out their conditions in the domain. It is affected by density divergence and multi-density behavior of data. To discuss these difficulties, the authors suggest an algorithm to the $\mathrm{x}$ value of one of the input parameters epsilon. AGED is called an automatic generation of multiple Eps [20]. It generates a set of values of Eps for several density levels that may exist in the dataset. Then, with many values of Eps, depending on the type of dataset either varied or non-varied, DBSCAN algorithm used for finding the clusters. The automatic generation of Eps points to better clustering with lesser efforts in the tuning of the same. The challenging subject is Eps estimation and approach of different density datasets. Intelligent Subspace Clustering [21] is a Density-based Clustering method approach of various density datasets of High Dimensional Datasets. AE-DBSCAN algorithm [22] introduce a novel method to determine the value of neighborhood radius Eps automatically. Yao et al. (2018)[23] highlight one; the noises directly in real highdimensional data have a non-Gaussian distribution with composite structures. They adjust Expectation Maximisation (EM) algorithm to determine on states of the parameters needed by the PMoG-LRR model. A Novel Parameter-Light Subspace Clustering Technique [24], is a subspace clustering method follows Single Linkage Method for numerical data called as CLUSLINK. The algorithm is following a single linkage clustering method and works in a bottom-up, greedy manner. Based on the knowledge of all parametric free clustering algorithms, the propose work constructed and formulated.

The next followed section describes the methodology of propose work.

\section{RESEARCH METHOD}

In this paper, propose a parameter-search method based on determining proper values of tuning parameters. And continue this proposal to covers multi densities clusters also. To handle the exercises to display the influence of input parameters in subspace clustering methods and to find out their proper values in the area. Empirical research confirms that the proposed strategy is consistent in different real-world datasets. 


\section{THE PROPOSED EFFICIENT-ENSUBCLU FLOW DIAGRAM:}

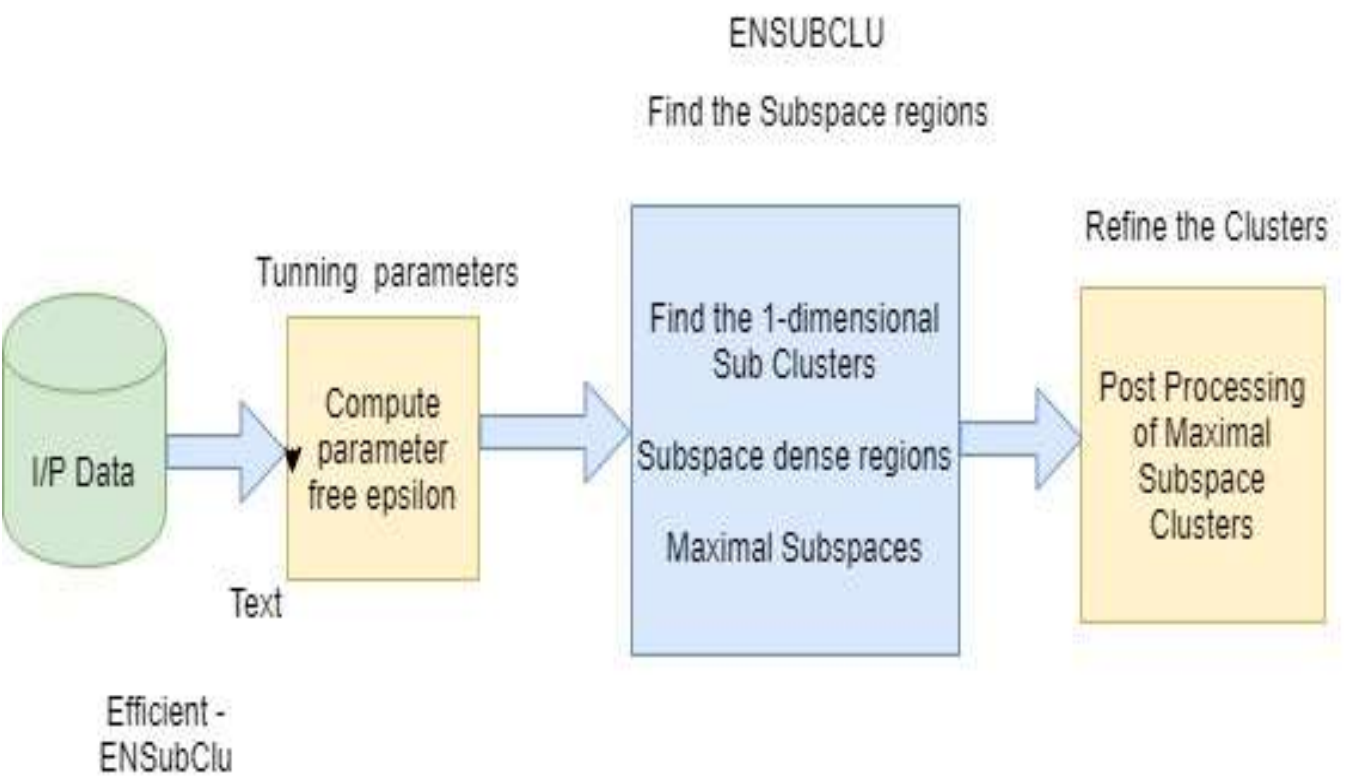

Fig. 3 Efficient EnSubClu Framework

\subsection{OVERVIEW OF EFFICIENT-ENSUBCLU PROCESSING}

This research paper designed an enhanced, Subspace Clustering framework model. The proposed Efficient-EnsubClu algorithm handles the density divergence problem, obtains maximal clusters, eliminates the redundant clusters and reduce the multiple database scans. An Efficient- EnSubClu model follows 4 phases.

First phase:

The density divergence problem is the major issue in high dimensional data clustering. Clusters densities vary in subspaces called the notion of density divergence. It means having different subspace cardinalities for different regions densities. To handle this drawback by discovering the clusters using different epsilon density thresholds in different subspaces.

$$
\text { Epsilon_Sub }{ }_{n}=\frac{\text { Minpoints } * \text { Maximum-distance_Sub }}{|\mathrm{DB}|}
$$

In this process, $n$-dimensional subspaces denoted by Sub_n while the sub $b_{n}$ is one among them.Maximum-distance_Subn defines the distance between the farther pair of

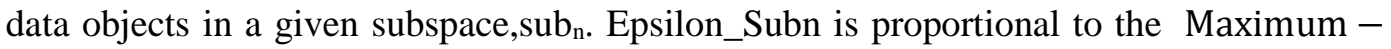
distance_Sub ${ }_{n}$ Which increases with the number of dimensions of the subspace. Min points are user deterministic input value provided to the proposed algorithm with which core point estimation is prepared. The total number of data objects defined in the dataset $|\mathrm{DB}|$. The clustering executes with epsilon input value computed automatically for interesting subspace using the equation mentioned above (1). The Epsilon_Subn is computed based on the spread of data objects either fixed Epsilon_Subn value or computed separately for each promising subspace refinements. To find the epsilon input value to avoid the density divergence problem. Moreover, constructing the onedimensional subspace dense units of a given dataset with epsilon $\varepsilon$, min points $\mu$ and discretization point $d_{\propto}$. 
Proposed Phase-1 Algorithm:

\section{Algorithm -1}

\section{/*Find the dense units in the one-dimensional data set*/}

1. Generate a $\mathrm{K}$ number of Unique numbers

2. For $\mathrm{i}=1$ to $\mathrm{K}$ do

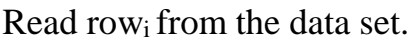

$$
\text { Assign } \mathrm{K}_{\mathrm{i}} \text { to row } \mathrm{i}
$$

//Assign $\mathrm{i}^{\text {th }}$ unique number to $\mathrm{i}^{\mathrm{t}}$ row

ArrayList_rowList<-row

ArrayList_dataList<-rowList.

End for;

3. For $i=1$ to $d$ //d-number of dimensions

For $\mathrm{j}=1$ to $\mathrm{k}$ $/ / \mathrm{k}$ no.of rows.

Read $\mathrm{i}^{\text {th }}$ element from the $\mathrm{j}^{\text {th }}$ row.

$$
\text { ArrayList_dlist }<-\mathrm{E}_{\mathrm{ij}} \quad / / \mathrm{E} \text { is actual data value. }
$$

End For;

Sort(dlist).

Dunit<-Find DenseUnits(dlist); $\quad$ //Find dense units by using

Write dunit to a file //dat file

End For;

\section{Algorithm -2}

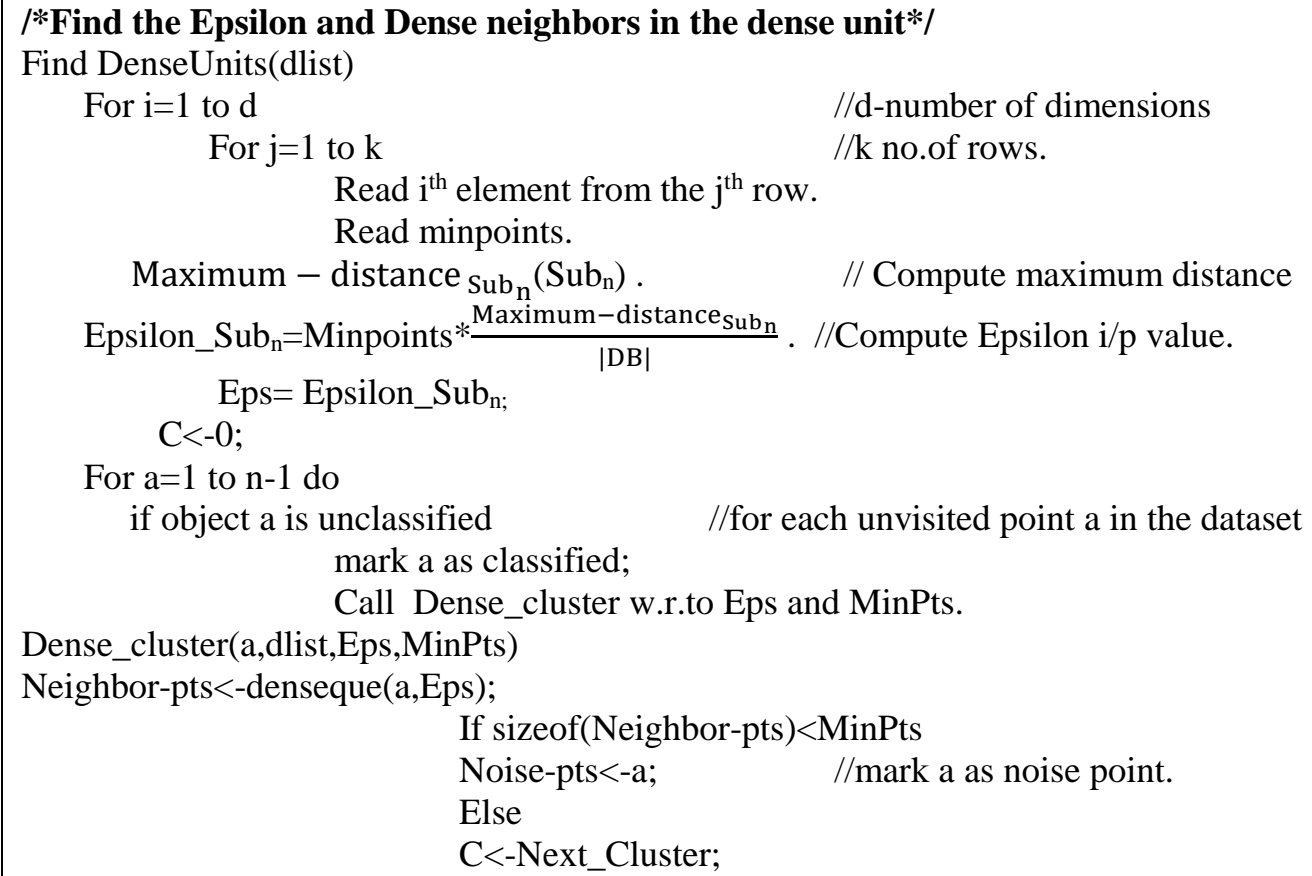




\section{Add a to cluster $C$. \\ For each point $\mathrm{a}^{1}$ is in Neighbor-pts \\ If $\mathrm{a}^{1}$ is unclassified;}

mark $\mathrm{a}^{1}$ as classified;

If sizeof(Neighbor-pts $\left.{ }^{1}\right)>=$ MinPts;

Neighbor-pts<-Neighborpts joined with

Neighborpoints.

Add $\mathrm{a}^{1}$ to cluster $\mathrm{C}$.

If $\mathrm{a}^{1}$ is not in cluster $\mathrm{C}$ then

End for:

End For.

The second phase follows the ENSUBCLU Model [6]process the steered subspace scheme on found one-dimensional subspaces. It follows the dense core regions of points in each of the one-dimensional subsets of dimension set of input datasets. To identify the steered common dense points from one-dimensional dense core region sets in higher dimensional subspace projections. Apply the steer jump approach to find all combinational dense core regions from all lower dimensions of dense units and considered in figure1. Moreover, it reduces the redundant subspaces using Hash data structure. (Merge dense neighbors).

\section{Algorithm -3}

\section{/*Generating of Maximal Subspace clusters*/ \\ Input:datfile. \\ Output: Cluster (DataMatrix file)}

1. Read LowNum, HighNum, Partition Size from the dat file.

2. Read dense units from the dat file

For $\forall$ dense unit items, dunit do

Adding entries $\{\operatorname{Sum}($ dunit), dunit $\}$ to DUS //DUS is assigned to store dense unit items

End For;

3. For $\mathrm{i}=1$ to $\mathrm{d}$

Read the dunit

For $\mathrm{j}=1$ to $\mathrm{n}$

element.

Get the dunit (Dense Unit Sum) of the dunit $_{\mathrm{j}}$;

DUS $<-\operatorname{dunit}(\mathrm{j})$ For $\forall$ DUS $=\{$ Sum, dunit $\} \in D U S$

//Get candidate dense items in $\mathrm{j}$ dimensions.

If indexkey of hash table DUS_hTable.Hash_T==Sum then //DUS_hTable contains DUS ${ }_{\mathrm{ij}}$

Adding i dimension to subspace Hash_T.S

//DUS $\mathrm{S}_{\mathrm{ij}}<$-append $\mathrm{i}$ to the value

Else

Adding new enteries(Sum,dunit,i) to DUS_hTable

$$
\text { //Add key<-DUS } \mathrm{ij}_{\mathrm{ij}} \text {,Value }<-\mathrm{i} \text { to the }
$$

DUS_hTable

End if

End For;

End For; 
Third, refines the maximal subspaces and find clusters in each found subspaces.

Follow the Algorithm -2 on each found subspace.

In phase 4: The research work proposes a new strategy is post-processing step to overcome the computational complexity connected with the DBSCAN by sufficient implementing new merging criteria at the initial stage of evaluation of clusters. That is applying the post-processing step on initial clusters. Calculate the distance of two clusters is $\mathrm{C} 1, \mathrm{C} 2$. If the distance $(\mathrm{C} 1, \mathrm{C} 2)<=$ Epsilon then merge the clusters.

1. Distance between Clusters is defined

$$
\operatorname{Dist}\left(C_{1}, C_{2}\right)=\operatorname{Min}\left\{\mathrm{d}(\mathrm{p}, \mathrm{q}): \mathrm{p} \in C_{1}, q \in C_{2}\right\}
$$

2.If $\operatorname{Dist}\left(C_{1}, C_{2}\right)<=\in$ then

Merge the clusters $C_{1}, C_{2}$.

3. Similar Clusters are merged as per equation(2)

4. Repeat the above steps till all small clusters are merged.

In addition to compute the number of subspace clusters in respective dimensions and the mean dimensionality of subspace clusters. It describes each cluster with less number of dimensions as evident from the low value of mean dimensionality.

$$
\text { Mean dimensionality }=\frac{\sum_{i=1}^{d} N_{i} * i}{\sum_{i=1}^{d} N}
$$

Where $N_{i}$ is the set of subspace clusters defined in the i-dimensional subspace.

Hence, we can obtain knowledge more concisely with an enhanced quality of clusters in terms of Accuracy and silhouette Coefficient[26][27].

Accuracy is one quality measure to obtain the purity of clusters and judge the quality.

$$
\text { Accuracy }=\left|\frac{\text { Correctly predicted objects }}{\text { All objects in the dataset }}\right|
$$

The quality of enhanced subspace clustering is analyzed interms of silhouette, which applies to unsupervised datasets[6][25].

$$
\text { The Silhouette } \operatorname{Index}=\operatorname{SCI}\left(x_{i}\right)=\frac{b_{i}-a_{i}}{\max \left(a_{i}, b_{i}\right)}
$$

Where

$$
\begin{array}{r}
a_{i}=\frac{1}{\left|C\left(x_{i}\right)\right|} \sum_{x_{j} \in C_{\left(x_{i}\right)}} \operatorname{dist}\left(x_{i}, x_{j}\right) \\
\text { and } b_{i}=\min _{C_{k} \in C /\left(x_{i}\right)} \sum_{x_{j} \in C_{k}} \frac{\operatorname{dist}\left(x_{i}, x_{j}\right)}{\left|C_{k}\right|}
\end{array}
$$

The approach suggested in this paper due to the performance of specific $\varepsilon$ for a particular cluster, can recognize clusters of various densities and can determine the density divergence of modern enhanced density-based subspace clustering techniques like ENSUBCLU, SUBSCALE in the event of multi-density clusters.

\section{RESULTS AND DISCUSSIONS}

In this section, we give a general evaluation of Efficient-EnSubClu. We executed Efficient-EnSubClu as well as the two enhanced steps Parametric free and meandimensionality of clusters. We evaluated Efficient-EnSubClu using several real-world and synthetic datasets. In all experiments, Efficient-EnSubClu could generate interesting maximal subspace clusters hidden in the high dimensional data.

The proposed algorithm Efficient-EnSubClu organized to formulate the relevant subspace clusters on various real-world data sets. Efficient-EnSubClu model implemented in Java language on 64 bit Windows 7 OS with Intel core i5-2600 and 8 GB 
RAM. Exercise synthetic datasets and real-world data sets from the UCI repository system [28] to the assessment of quality maximal subspaces. The performance of Efficient-EnSubClu in terms of efficiency and accuracy on a series of experiments. Analyze the efficiency scalability, and the accuracy of Efficient-EnSubClu against existing well-known density based subspace clustering algorithms are ENSUBCLU[6], SUBSCALE[16], EDENCOS[17], SUBCLU[5]. Examine the accuracy, Silhouette coefficient(SCI) [26][27] of Efficient-EnSubClu model with the following SUBSCALE, subspace clustering method on Small soyabean(47 X 34) dataset with various minpoints as parameter, which are represented in table 1.

In this section, it is explained the results of research and at the same time is given the comprehensive discussion. Results can be presented in figures, graphs, tables and others that make the reader understand easily $[2,5]$. The discussion can be made in several subchapters.

Table I. Comparison of Eff-EnSubClu and Subscale interms of \#Subspace Clusters, Purity SCI for Small soyabean(47 X 34)

\begin{tabular}{|c|c|c|c|c|c|c|}
\hline & \multicolumn{2}{|l|}{ Eff-EnSubClu } & SUBSCALE & \multicolumn{2}{l|}{} \\
\hline $\begin{array}{l}\text { Min } \\
\text { points }\end{array}$ & $\begin{array}{l}\text { \# SubSpace } \\
\text { Clusters }\end{array}$ & Accuracy & SCI & $\begin{array}{l}\# \\
\text { SubSpace } \\
\text { Clusters }\end{array}$ & Accuracy & SCI \\
\hline 2 & 30178 & 0.952 & 0.68 & 39178 & 0.941 & 0.58 \\
\hline 3 & 37564 & 0.943 & 0.65 & 46561 & 0.932 & 0.62 \\
\hline 4 & 41000 & 0.934 & 0.63 & 51077 & 0.921 & 0.61 \\
\hline 5 & 35000 & 0.912 & 0.61 & 45009 & 0.901 & 0.59 \\
\hline
\end{tabular}

Examine the algorithms, Eff- EnSubClu and SUBSCALE based on the number of subspace clusters obtained. By the increase in the value of min-points, the number of subspace clusters received Accuracy and Silhouette coefficient decreases continuously. Though, Eff-EnSubClu produces the less number of subspace clusters with improved Accuracy and Silhouette coefficient decreases compared to SUBSCALE. Figure 4 shows the corresponding chart to plot the number of subspace clusters upon improved values of min-points, for Soya bean dataset [28].

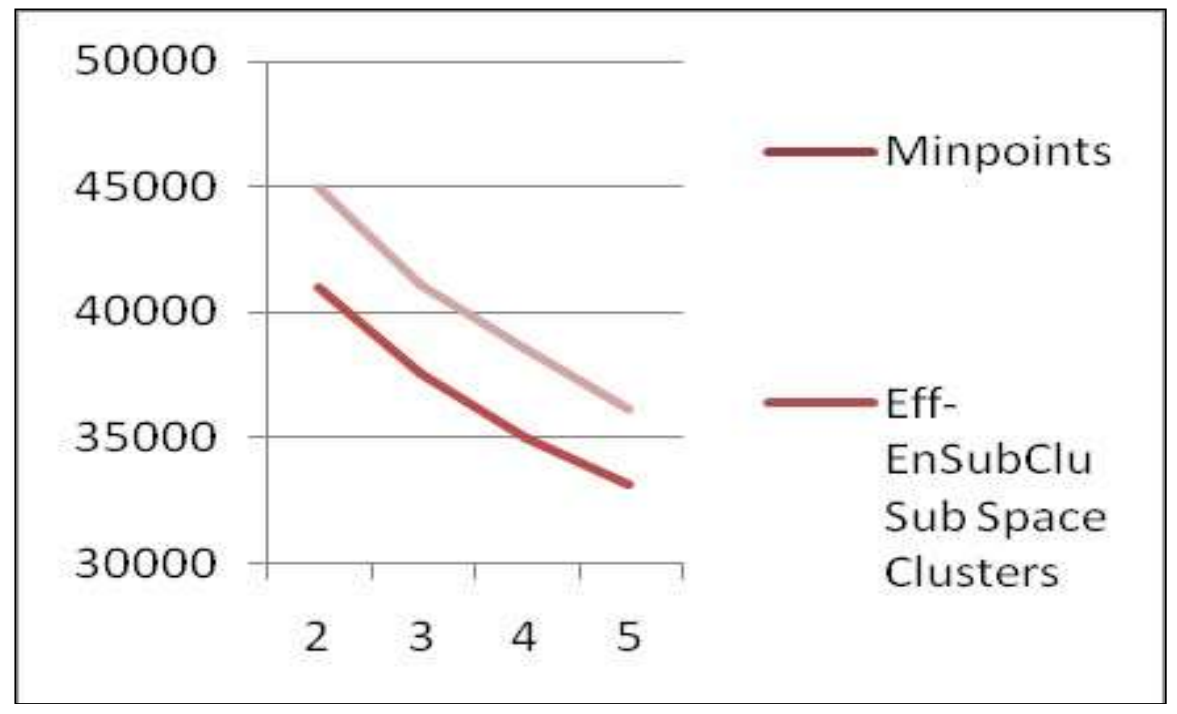

Fig. 4 Comparison of subspace clusters obtained with increase in Minpoints 
The mean - dimensionality and number of subspace clusters of all suggested algorithms on a series of real-world data sets. The following Fig 5 represents the Silhouette Coefficient values(SCI) of Eff-EnSubClu, SUBSCALE, ENSUBCLU and EDENCOS Subspace clustering algorithms on soyabean (47 X 34) data set. Observed that, the Eff-EnSubClu is effective SCI values than other subspace clustering methods with various minpoint parameters.

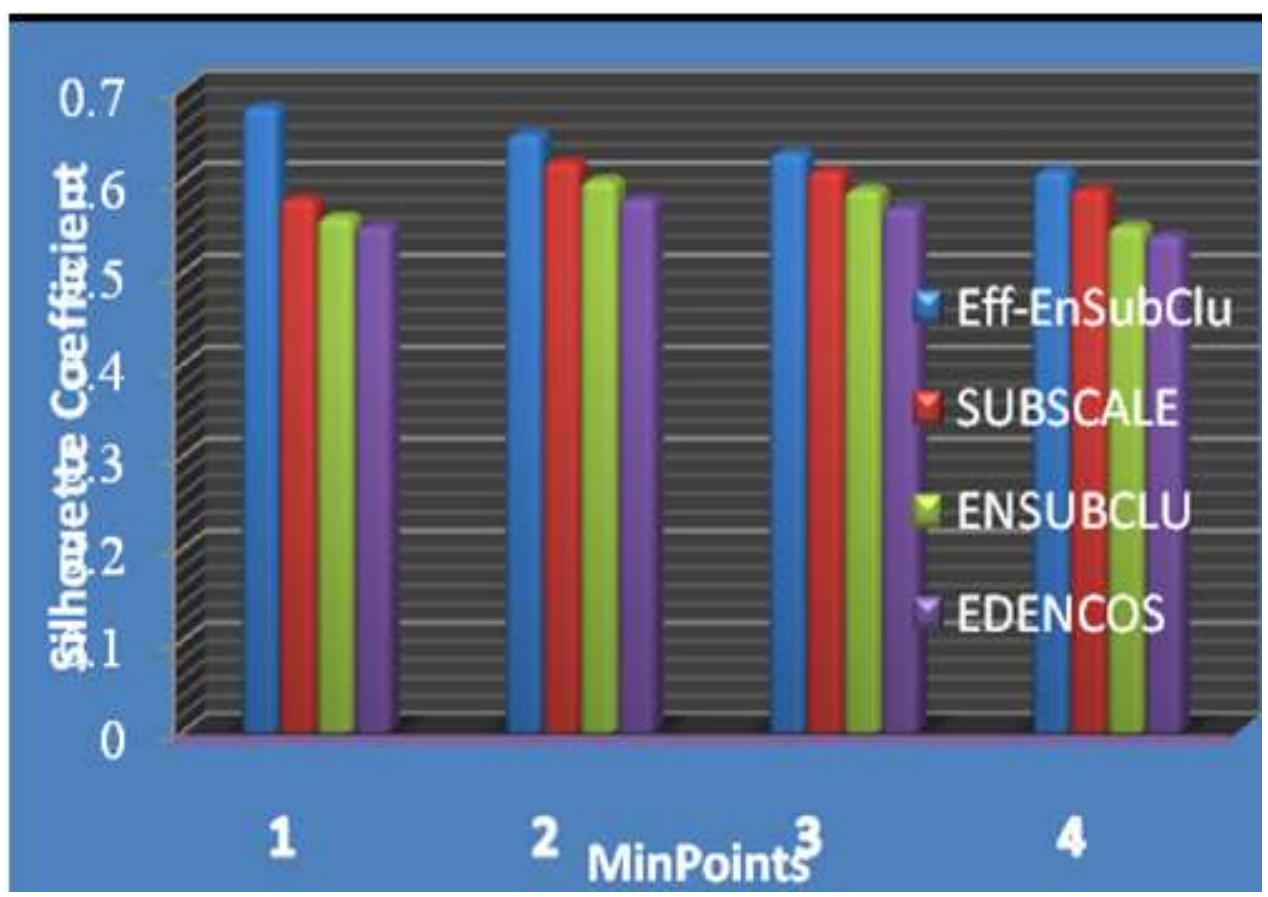

Fig. 5 Comparsion of Silhouette coefficient (SCI) values of mentioned algorithims for Soyabean dataset

The mean - dimensionality and number of subspace clusters of all suggested algorithms on a series of real-world datasets. The number of Subspace Clusters, Mean dimensionality of Subspace Clusters for the growing of data dimensionality on real world datasets represented in the following table II.

Table II. Comparison of Efficient-EnSubClu, SUBSCALE and EDENCOS interms of \#Subspace Clusters, Mean dimensionality of Subspace Clusters for Real world datasets.

\begin{tabular}{|l|c|c|c|c|c|c|}
\hline \multirow{2}{*}{$\begin{array}{c}\text { Real world } \\
\text { Datasets }\end{array}$} & \multicolumn{2}{|c|}{ Efficient - EnSubClu } & \multicolumn{2}{c|}{ SUBSCALE } & \multicolumn{2}{c|}{ EDENCOS } \\
\cline { 2 - 6 } & $\begin{array}{c}\text { Subspac } \\
\mathrm{e} \\
\text { Clusters } \\
\text { dimensionality } \\
\text { of Subspace } \\
\text { Clusters }\end{array}$ & $\begin{array}{c}\text { Subspace } \\
\text { Clusters }\end{array}$ & $\begin{array}{c}\text { Mean } \\
\text { dimensional } \\
\text { ity of } \\
\text { Subspace } \\
\text { Clusters }\end{array}$ & $\begin{array}{c}\text { Subspace } \\
\text { Clusters }\end{array}$ & $\begin{array}{c}\text { Mean } \\
\text { dimensionality } \\
\text { of Subspace } \\
\text { Clusters }\end{array}$ \\
\hline Face (50X2250) & 34178 & 2.152 & 49178 & 2.941 & 53266 & 3.441 \\
\hline Soyabean (47x34) & 47564 & 1.943 & 49567 & 2.332 & 63456 & 4.932 \\
\hline Wages(11x534) & 31009 & 2.934 & 41075 & 3.121 & 76558 & 3.921 \\
\hline Diabetes(8X768) & 33657 & 1.912 & 39566 & 2.501 & 54683 & 2.801 \\
\hline
\end{tabular}


The following figure 6 refers the Diabetes $(8 \times 768)$ dataset to generation of number of subspace clusters with mean dimensionality of subspace clusters comparisons in various subspace clustering models with proposed Efficient-EnSubClu model better results. Hence the better algorithm, that results in clustering solution with low mean dimensionality is preferred. It is found tha less mean dimensionality of subspace clusters produced by Efficient-EnSubClu model.

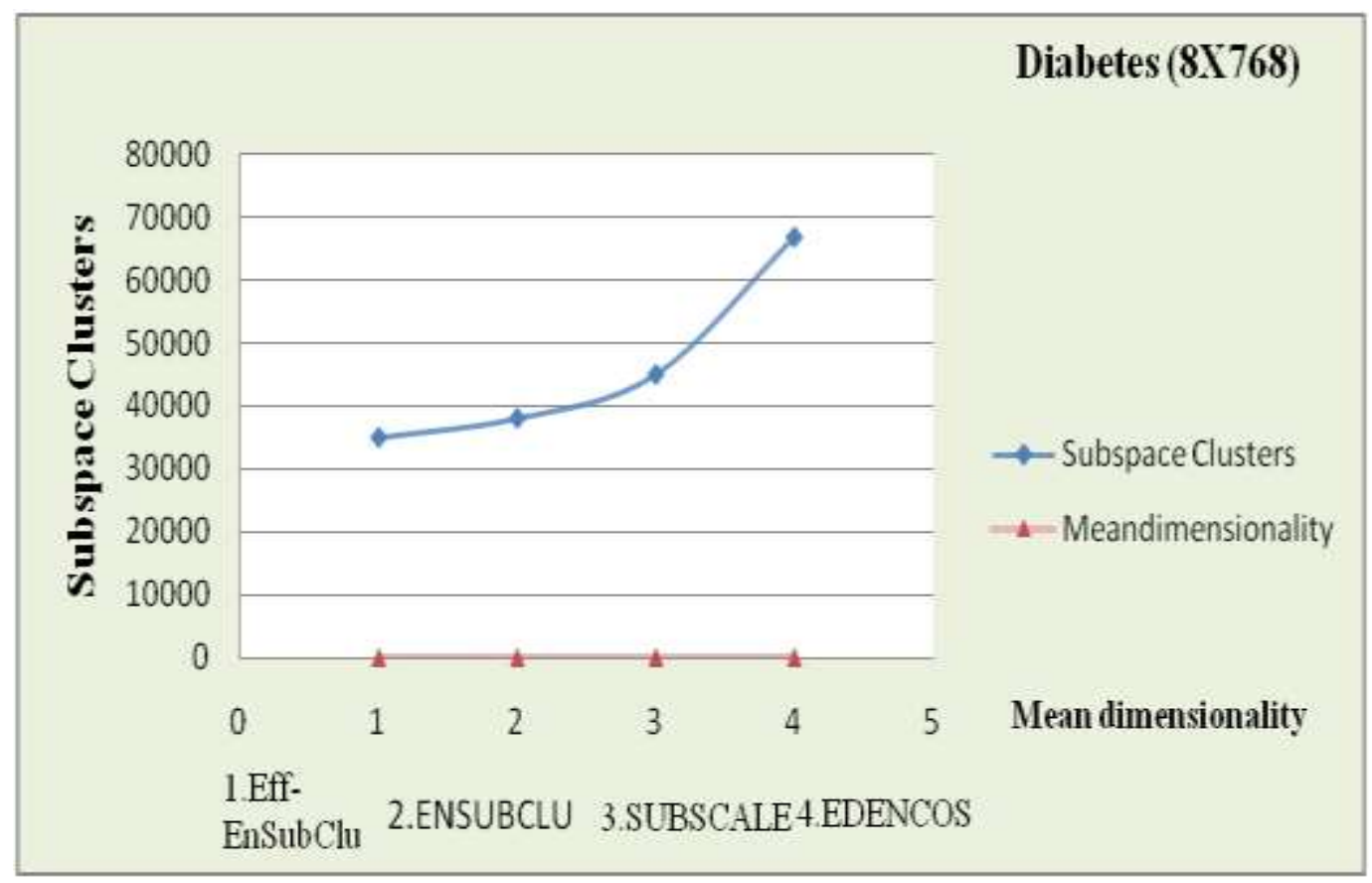

Fig. 6 Comparison of subspace clusters obtained with Mean dimensionality

\section{CONCLUSION}

Overall, the research presented in this paper provides many insights into theoretically and empirically characterizing the problem of enhanced subspace Clustering. In this paper, we first motivated the need for a tunning parameter-free method To estimate Epsilon and avoid the density Divergence problem in Efficient Enhanced Subspace Clustering. By defining the epsilon $\epsilon$, distance parameter dynamically furthermore adaptively at a specific attribute level, it allows for incremental learning by providing qualifying parameter settings adaptively. This method leads to better cluster generation at higher dimensionality. Later apply the approach ENSUBCLU (Enhanced Subspace Clustering) which uses the density-based clustering to find Subspace Clusters installed in higher dimensional clusters. A new strategy is post-processing step to associate with the DBSCAN by efficient implementing new merging criteria at the initial stage of evaluation of clusters Post-processing steps applied on each found subspaces. Finally, This model is expected to be useful in solving data mining problems in many applications. The significant performance and quality of cluster results of a subspace clustering algorithm are very conditioned on the parameter values input to the algorithm. Efficient-EnSubClu is implemented and tested on various benchmark and real and synthetic data sets. It outperforms ENSUBCLU, SUBSCALE, EDENCOS algorithms in terms of cluster quality and execution time. In future, this could be extended for all kinds of data and Estimate the different correlation measures to identifying the correlated subspaces instead of distance similarity measures. 


\section{REFERENCES}

[1] Ester, Martin, et al. "A density-based algorithm for discovering clusters in large spatial databases with noise." Kdd. Vol. 96. No. 34. 1996.

[2] Agrawal, Rakesh, et al. Automatic subspace clustering of high dimensional data for data mining applications. Vol. 27. No. 2. ACM, 1998.

[3] Sim, Kelvin, et al. "A survey on enhanced subspace clustering." Data mining and knowledge discovery 26.2 (2013): 332-397

[4] Devi, J. Rama, and M. Venkateswara Rao. "An era of Enhanced Subspace Clustering in HighDimensional data." i-Manager's Journal on Computer Science 4.3 (2016): 29.

[5] Kailing, Karin, Hans-Peter Kriegel, and Peer Kröger. "Density-connected subspace clustering for highdimensional data." Proceedings of the 2004 SIAM international conference on data mining. Society for Industrial and Applied Mathematics, 2004.

[6] J. RAMA DEVI, Dr. M.VENKATESWARA RAO, "A new generic interpretation of enhanced subspace clustering in high dimensional data." International Journal of Engineering \& Technology, v. 7, n. 4, pp.4157-4163, dec. 2018. ISSN 2227-524X.

[7] Cheng, Chun-Hung, Ada Wai-Chee Fu, and Yi Zhang. Entropy-based subspace clustering for mining numerical data. Diss. Chinese University of Hong Kong, 1999.

[8] Lakshmi, B. Jaya, K. B. Madhuri, and M. Shashi. "An efficient algorithm for density based subspace clustering with dynamic parameter setting." Int. J. of Information Technology and Computer Science (IJITCS) 6 (2017): 27-33

[9] Goil, Sanjay, Harsha Nagesh, and Alok Choudhary. "MAFIA: Efficient and scalable subspace clustering for very large data sets." Proceedings of the 5th ACM SIGKDD International Conference on Knowledge Discovery and Data Mining. Vol. 443. ACM, 1999.

[10] Yadav, Jyoti, and Dharmender Kumar. "Sub space Clustering using CLIQUE: an exploratory study." Int. J. Adv. Res. Comput. Eng. Technol. (IJARCET) 3, (2014).

[11] Chu, Yi-Hong, et al. "Density conscious subspace clustering for high-dimensional data." IEEE Transactions on knowledge and data engineering 22.1 (2008): 16-30.

[12] Kriegel, H-P., et al. "A generic framework for efficient subspace clustering of high-dimensional data." fifth IEEE international conference on data mining (ICDM'05). IEEE, 2005.

[13] Assent, Ira, et al. "DUSC: Dimensionality unbiased subspace clustering." seventh IEEE international conference on data mining (ICDM 2007). IEEE, 2007.

[14] Müller, Emmanuel, et al. "Scalable density-based subspace clustering." Proceedings of the 20th ACM international conference on Information and knowledge management. ACM, 2011.

[15] Assent, Ira, et al. "INSCY: Indexing subspace clusters with in-process-removal of redundancy." 2008 Eighth IEEE International Conference on Data Mining. IEEE, 2008.

[16] Kaur, Amardeep, and Amitava Datta. "Subscale: fast and scalable subspace clustering for high dimensional data." 2014 IEEE International Conference on Data Mining Workshop. IEEE, 2014.

[17] Palaniammal, S. "Improved subspace clustering algorithms for high dimensional data." (2016).

[18] Lakshmi, B. Jaya, M. Shashi, and K. B. Madhuri. "A rough set based subspace clustering technique for high dimensional data." Journal of King Saud University-Computer and Information Sciences (2017).

[19] Lee, Dongjin, and Junho Shim. "Impact parameter analysis of subspace clustering." International Journal of Distributed Sensor Networks 11.9 (2015): 398452.

[20] Soni, Neha. "Aged (automatic generation of eps for dbscan)." International Journal of Computer Science and Information Security 14.5 (2016): 536.

[21] Sunita Jahirabadkar, and Parag Kulkarni, Intelligent Subspace Clustering, A Density based Clustering aproach for High Dimensional Dataset, World Academy of Science, Engineering and Technology, International Journal of Computer and Information Engineering, Vol:3, No:7, 2009

[22] Ozkok, Fatma Ozge, and Mete Celik. "A new approach to determine Eps parameter of DBSCAN algorithm." International Journal of Intelligent Systems and Applications in Engineering5.4 (2017): 247-251.

[23] Yao, Jing, et al. "Robust subspace clustering via penalized mixture of Gaussians." Neurocomputing 278 (2018): 4-11.

[24] Kelkar, Bhagyashri A., Sunil F. Rodd, and Umakant P. Kulkarni. "A Novel Parameter-Light Subspace Clustering Technique Based on Single Linkage Method." Journal of Information \& Knowledge Management 18.01 (2019): 1950007.

[25] Inturi, Anitha Rani, and Jujjuri Ramadevi. "An Unsupervised Approach for Mining Multiple Web Databases." International Journal of Electronics and Computer Science Engineering, Jg1: 2148-2151.

[26] Rousseeuw, Peter J. "Silhouettes: a graphical aid to the interpretation and validation of cluster analysis." Journal of computational and applied mathematics 20 (1987): 53-65.

[27] Ramadevi Jujjuri, Dr.M.V Rao, Evaluation of Enhanced Subspace Clustering Validity Using Silhouette Coefficient Internal Measure, Journal of Advanced Research in Dynamical \& Control Systems, Vol. 11, No. 1, 2019.

[28] Lichman, UCI Machine Learning Repository, available at 2007.http://archive.ics.uci.edu/m/. 
International Journal of Advanced Science and Technology Vol.128 (2019) 